\title{
The european FAZIA initiative: a high-performance digital tele- scope array for heavy-ion studies
}

\author{
G. Casini ${ }^{1}$,a, S. Barlini ${ }^{1}$, G. Pasquali ${ }^{1}$, G. Pastore ${ }^{1}$, M. Bini ${ }^{1}$, S. Carboni ${ }^{1}$, A. Olmi ${ }^{1}$, S. Piantelli ${ }^{1}$, \\ G. Poggi ${ }^{1}$, A. Stefanini ${ }^{1}$, S. Valdré ${ }^{1}$, E. Bonnet ${ }^{3}$, B. Borderie ${ }^{4}$, R. Bougault ${ }^{5}$, M. Bruno ${ }^{2}$, A. Chbihi ${ }^{3}$, \\ M. Cinausero ${ }^{6}$, M. Degerlier ${ }^{8}$, P. Edelbruck ${ }^{4}$, J.D. Frankland ${ }^{3}$, F. Gramegna ${ }^{6}$, D. Gruyer ${ }^{3}$, \\ M. Guerzoni' ${ }^{2}$, A. Kordjasz ${ }^{9}$, T. Kozik ${ }^{10}$, N. Le Neindre ${ }^{5}$, O. Lopez ${ }^{5}$, T. Marchi6,7, P. Marini ${ }^{3}$, \\ L. Morelli ${ }^{2}$, A. Ordine ${ }^{11}$, M. Pârlog ${ }^{5}$, M.F. Rivet ${ }^{4}$, E. Rosato ${ }^{11}$, F. Salomon ${ }^{4}$, G. Spadaccini ${ }^{11}$, \\ T.Twaróg ${ }^{9}$, E. Vient ${ }^{5}$, and M. Vigilante ${ }^{11}$
}

${ }^{1}$ INFN, Sezione di Firenze and Dipartimento di Fisica, Università di Firenze, Italy

${ }^{2}$ Dipartimento di Fisica, Università di Bologna and INFN, Sezione di Bologna, Italy

${ }^{3}$ GANIL, CEA/DSM-CNRS/IN2P3, B.P. 5027, F-14076 Caen cedex, France

${ }^{4}$ Institut de Physique Nucléaire, CNRS/IN2P3, Université Paris-Sud 11, F-91406 Orsay cedex, France

${ }^{5}$ LPC, IN2P3-CNRS, ENSICAEN et Université de Caen, F-14050 Caen-Cedex, France

${ }^{6}$ INFN, Laboratori Nazionali di Legnaro, Padova, Italy

${ }^{7}$ Dipartimento di Fisica, Università di Padova, Italy

${ }^{8}$ Nevsehir University, Science and Art Faculty, Physics Department, Nevsheir, Turkey

${ }^{9}$ Jagiellonian University, Institute of Nuclear Physics IFJ-PAN, PL-31342 Kraków, Poland.

${ }^{10}$ Warsaw University, Poland

${ }^{11}$ Dipartimento di Fisica, Università Federico II di Napoli and INFN, Sezione di Napoli, Italy

\begin{abstract}
The european Fazia collaboration aims at building a new modular array for charged product identification to be employed for heavy-ion studies. The elementary module of the array is a Silicon-Silicon-CsI telescope, optimized for ion identification including pulse shape analysis, too. The achievement of top performances imposes specific electronics which has been developed by the FAZIA collaboration and includes high quality charge and current preamplifiers, coupled to fully digital front-end. During the initial R\&D phase, original and novel solutions have been tested in prototypes, obtaining unprecedented ion identification capabilities. FAZIA is now constructing a demonstrator array consisting of about two hundreds telescopes arranged in a compact and transportable configuration. In this contribution, we mainly summarize some aspects studied by FAZIA to improve the ion identification. Then we will briefly discuss the FAZIA program focused on experiments to be done with the demonstrator. First results on the isospin dynamics obtained with a reduced set-up demonstrate well the performance of the telescope and represent a good starting point towards future investigations with both stable and exotic beams.
\end{abstract}

\footnotetext{
a e-mail: casini@fi.infn.it
} 


\section{EPJ Web of Conferences}

\section{Introduction}

The studies of the reaction mechanisms occurring in heavy ion collisions have been recently focused on observables related to the isospin ${ }^{1}$ content of the system. Such observables allow to access the isovector term of the nuclear potential, whose behaviour is poorly known for nuclei far from groundstate conditions. In particular, the density and the temperature dependence of the nuclear symmetry energy $\left(E_{\text {sym }}\right)$ are the subject of many recent theoretical [1-3] and experimental [4-7] studies, which investigated heavy-ion reactions at different regimes of bombarding energy, impact parameter and system size. Since a common feature of most collisions is the production of multiparticle final states, a crucial experimental challenge is to detect and recognize at the best the many reaction products, as a preliminary necessary step to trace back to the interaction phases [8]. Recently, the detector frontier has become the isotopic identification of fragments in a wide range of kinetic energies and over large solid angles [9-11]. Keeping this in mind, some years ago the european FAZIA collaboration was born from a previous cooperation among groups involved in this research field at several laboratories. FAZIA started a research program to optimize the detectors in terms of isotopic identification and, after that, to construct a modular array with state-of-the-art performances that will be used for heavyion experiments with both stable and radioactive ion beams, such as those to be delivered by SPES (LNL), SPIRAL2 (GANIL) and FRIBS (LNS) facilities.

\section{The FAZIA module}

In order to build a versatile and transportable array for charged fragments the FAZIA project is based on a conventional solid-state telescope as done by other groups [10,12]. The telescope (active area $20 \times 20 \mathrm{~mm}^{2}$ ) is made of two Silicon layers ( 300 and $500 \mu \mathrm{m}$ thick, respectively) followed by a slightly tapered CsI(Tl) scintillator $100 \mathrm{~mm}$ long. As usual, this device allows one to separate particles which punch through a first active layer by means of the well known $\Delta E-E$ technique, where $E$ is the energy released in the second layer. To increase the dynamical range of the telescope (FAZIA is supposed to work from 10 to $50 \mathrm{MeV} / \mathrm{u}$ ), three detectors are used so that the $\Delta E-E$ method can be applied using more signal combinations. In the $\Delta E-E$ method the energy threshold for ion identification ( $\mathrm{Z}$ and/or A) is simply the minimum energy required to punch through the $\Delta E$ layer. A strong effort was done by FAZIA to improve the performance (separation capability and threshold) while maintaining good energy information. Therefore, particular care was devoted to verify the limits of identification for particles stopped in the first silicon, basing on the Pulse Shape Analysis (PSA). Accurate PSA strongly benefits from digital fast sampling electronics coupled to high quality analog stages, which preserve the original waveforms. Indeed, FAZIA complemented the R\&D on the detectors with the construction of high-performing electronic channels, each made of a low-noise preamplifier with current and charge outputs, followed by complex stages where the signals are sampled and processed by FPGA to extract the needed information. Such a concept has been implemented in the test phases on single channel electronics, while for the demonstrator array an integrated multifunction FEE equipment has been developed which serves many channels and operates under vacuum.

The results obtained by FAZIA are described in many papers (see e.g. [13-18]) which we refer to. In this Section we collect an anthology of these results to enlighten some critical aspects, the proposed solutions and the obtained performance. Moreover, we introduce some preliminary results on the PSA in partially depleted silicons emerging from the most recent tests.

\footnotetext{
${ }^{1}$ we adopt the widespread usage of isospin for the $\mathrm{N} / \mathrm{Z}$ ratio
} 
INPC 2013

\subsection{Channeling effects}

Ion identification via PSA in silicon is critically sensitive to spurious sources which modify the native (charge or current) waveforms. Channeling effects are one of these sources as clearly demostrated by FAZIA with specific experiments, where a telescope was remotely and finely rotated under irradiation to select or avoid specific crystallographic directions $[13,15]$. The performance of the detector strongly degrades for orientations close to the crystal principal planes or axes: it was found that energy and charge risetime resolutions worsen by a factor of three with respect to random orientations. The effect is large not only for PSA but also for the conventional $\Delta E-E$ method. Accordingly, FAZIA silicons are made from wafers cut in such a way that the ions hit them along 'random' paths (about $7^{\circ}$ off the $<111>$ direction) all over the active area.

\subsection{Preserving original signal waveforms for PSA}

The time development of signals in particle detectors depends on the drift electric field. Hence, for PSA applications, it is important to avoid any variation of the applied electric field with time and impact position on the detector, as pointed out years ago [19]. FAZIA examined this subject using the powerful tools offered by digital electronics and by versatile supply commercial equipments. To reduce variations with time, FAZIA developed a specific bias supply system with a feedback loop which measures the current during the experiment and adjusts the applied voltage to compensate the drop across the bias resistor.

Concerning the effects due to silicon doping, FAZIA studied the quality of ion identification in silicons with different inhomogeneities of dopant concentration. Indeed, for a given applied junction voltage, the effective electric field is a function of the bulk resistivity (i.e. of the doping) which is therefore important to keep as constant as possible. With specific tests [15], we demonstrated the need to keep the inhomogeneities below a few percent for good PSA applications. No effect, as expected, has been found on the $\Delta E-E$ method. Hence, the present FAZIA recipe requests the use of silicons with high doping homogeneities, such as those attained nowadays through neutron transmutation techniques. Our typical silicons have a resistivity $\rho=3000-4000 \Omega \cdot \mathrm{cm}$. To verify in a rather easy way, without beam, the doping homogeneity of silicon crystals, a procedure based on a UV-laser has been implemented [14], which allows us to produce a resistivity map for each detector before its final assembling. We finally mention that PSA is also spoiled by fluctuations induced by radiation damage effects. The final effect of several types of damage produced in silicon by impinging ions results in microscopic variations of the electric field and/or favour electron-hole recombination or trapping. As a result, fluctuations of signal shapes increase and PSA is degraded. Such effects, largely dependent on the ion charge and energy, are difficult to summarize and to schematize; a specific experiment to study how PSA degrades with ion fluence has been done using Xe ions at $35 \mathrm{MeV} / \mathrm{u}$ [18].

All the above reported effects causing signal shape variations are critical for PSA, especially when one aims at some isotopic separation which is associated to weak variations of the signal shape. In this respect, once all these spoiling contributions have been kept under control (from silicon production and wafer cut to electronics performances), one can reach a lower threshold for good [16] mass separation $\sim 5 \mathrm{MeV} / \mathrm{u}[13]$ in the region of Carbon ions.

\subsection{Front and rear irradiation}

Since the pioneer works on PSA with silicon detectors [20], it was argued that reverse mounting operation would improve ion identification thanks to the overall lengthening of signal risetimes when ions enter the ohmic side of the device. The choice of FAZIA, from the very beginning, was indeed 
to adopt the reverse configuration. Possible drawbacks of this choice are worse timing and energy resolutions, especially for slow particles stopped in the very low electric field region. As a matter of fact, in most silicon arrays people adopted the popular "front" configuration with the junction (high field) side facing incoming ions. Recently, thanks to digital electronics and with the goal to lower ion identification thesholds, the mounting geometry of silicon detectors has been reconsidered [11, 21] in order to compare the resolutions attainable in the two configurations, but conclusive tests were missing. FAZIA studied this aspect with a specific experiment where a given three-layer telescope featuring high quality silicon stages (as described in the previous sections) was used. The telescope was irradiated in the same controlled conditions (beam, target, distances and angles, bias voltage and electronic chain) but in two phases: first the silicon chips have been irradiated in "front" mounting, then they have been flipped and used for the rest of the measurement. A comparison of the two obtained data sets [17] showed that the $\Delta E-E$ technique gives the very same good resolution provided that sufficiently long shaping times are used for signal filtering (to avoid possible ballistic deficit). On the contrary, sizeable differences are found for PSA: front irradiation is definitely worse. Indeed, of the two employed shape related variables (plotted in bidimensional correlation with the deposited energy E), i.e. the charge signal risetime (QRT) and the maximum of the current signal (IMAX), only the first one gives $\mathrm{Z}$ identification in front mounting, while IMAX fails. In rear mounting, instead, particle are identified using both shape variables and, more important, the thresholds are also improved. By using the E-QRT plot, in front mounting geometry the $\mathrm{Z}$ separation is obtained for ions with range in silicon greater than $170 \mu \mathrm{m}(\mathrm{Z} \leq 10)$ to $250 \mu \mathrm{m}$ ( $\mathrm{Z} \sim 30$, zinc); in reverse mounting the thresholds for charge separation drop as low as 30-40 $\mu \mathrm{m}$ for $\mathrm{Z} \leq 8$, steadly increasing up to $150 \mu \mathrm{m}$ for Zinc ions.

\subsection{PSA in partially depleted silicons: preliminary results}

Preliminary results are reported here on a recent test to investigate the PSA in partially depleted silicon detectors. So far, the usual operating condition for the FAZIA silicons (as for other collaborations) corresponded to a moderate (10-20\%) overbias, in order to avoid a nominally zero electric field region and to reduce dead-layers. It was implicitely supposed that it is a bonus for typical performance to avoid phenomena due to an almost vanishing electric field, such as an extremely slow charge collection or even partial charge carrier deficit (in case of e-h recombination). However, at least for PSA, the longer time scales associated to signal formation could in principle increase the sensitivity and improve the ion separation. To study this aspect, FAZIA recently performed an experiment using a standard silicon telescope where the first $300 \mu \mathrm{m}$ silicon was kept at the usual voltage (slightly overbiased) while the following $500 \mu \mathrm{m}$ detector was operated at several voltages, from the nominal depletion $(290 \mathrm{~V})$ to a very low value $(105 \mathrm{~V})$, the latter corresponding to an undepleted layer (nominal zero field) of $200 \mu \mathrm{m}$; in this condition, this second detector could behave as a $300 \mu \mathrm{m}$ silicon with an initial undepleted (and possible inactive) layer. The first $\Delta E$ silicon is important as a reference to identify ions via the $\Delta E-E$ method and to calibrate the raw $E$ axis. Indeed, at underdepletion voltages one expects that the energy scale can vary with respect to the standard calibration, due to the presence of a variable dead-layer (we adopt rear injection mounting) and, as said, of recombination effects. So, some non-linarity is expected in the energy scale, also depending on the ion type and energy. The other expected effect is the increase of the signal risetimes; indeed, QRT values up to $10 \mu$ s have been found at $105 \mathrm{~V}$. This, in turn, can produce an apparent pulse height deficit if the shaping times are not long enough. Therefore, FAZIA extended in this experiment the memory depth for the sampled waveforms to very large values (70 $\mu \mathrm{s}$ ) thus suppressing any ballistic deficit. This long sampling duration was used along the measurement for all signals and for each voltage value. Some preliminary results of the data analysis (in progress) are listed below. 


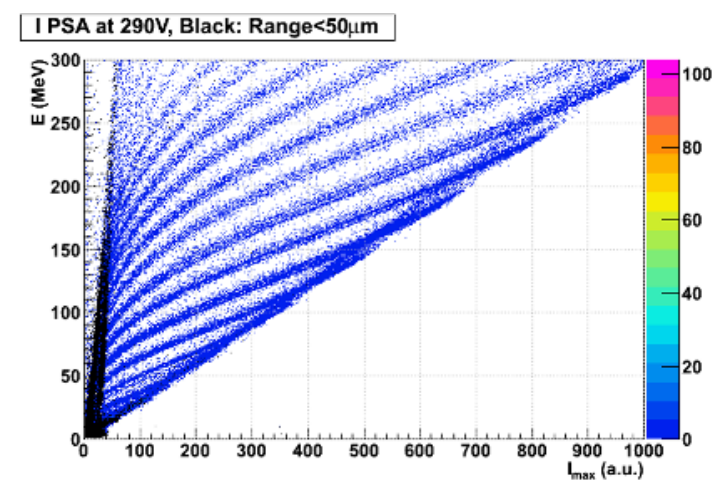

(a)

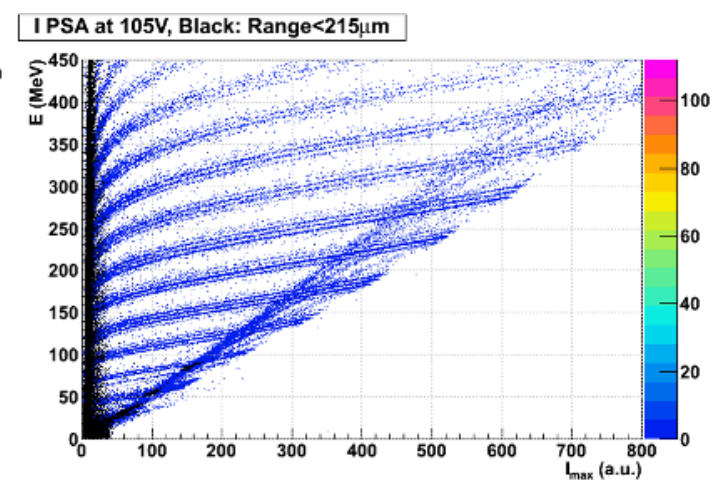

(b)

Figure 1: PSA identification plot of $\mathrm{E}$ as a function of IMAX for a $500 \mu \mathrm{m}$ FAZIA silicon operated at different voltages. Letf: full depletion voltage, $290 \mathrm{~V}$. Ions are separated in charge but not in mass. Black points are events of ions with a range shorter than $50 \mu \mathrm{m}$. Right: strong underdepletion voltage, $105 \mathrm{~V}$. Ions are separated in charge and in mass up to $Z \sim 10$. Black dots correspond to ions with range less than an arbitrary value of $215 \mu \mathrm{m}$. Some punching through events appear here, more clearly than at $290 \mathrm{~V}$. They survive the CsI(Tl) veto due to a imperfect geometrical match with the silicon pair.

1. About energy, with very long filtering, the non-linearities of the scale are unexpectedly small, even for the heaviest measured ions ( $\mathrm{Kr}$ beams were used) and even at the lowest bias voltage $(105 \mathrm{~V})$. In other words, the residual pulse height defect with respect to the full depletion is small (within 1-2\%) over a wide ion and energy domain. It seems that the supposed dead layer $(200 \mu \mathrm{m}$ at $105 \mathrm{~V})$ behaves as an active region. This can be associated to the funneling phenomenon [22], whose features are rather unexplored especially for energetic heavy ions.

2. According to the previous point, the capability of the telescope to identify ions with the $\Delta E-E$ technique is essentially preserved also for incomplete depletion, over all the measurable spectrum of fragments.

3. As expected, a huge increase of the signal timescale is observed at reduced voltage values. In the present case, charge signal risetimes increase from $400 \mathrm{~ns}$ at $290 \mathrm{~V}$ to over $10000 \mathrm{~ns}$ at $105 \mathrm{~V}$. Accordingly, the shapes of the correlations used for ion identification (E vs. QRT or E vs. IMAX) change a lot.

4. About PSA we refer to the Fig. 1. This test confirms (Fig. 1a) that good $\mathrm{Z}$ identification is reached at full depletion, with threshold values (around $50 \mu \mathrm{m}$ for $\mathrm{Z} \leq 10$ ) in agreement with previous findings $[16,17]$. Due to several not optimized aspects (reduced preamplifier sensitivity and non excellent doping homogeneity) only a marginal isotopic separation is observed for the lightest fragments (up to oxygen or so) in this condition. When decreasing the voltage, one sees an increase of the energy limit for ion separation: at $105 \mathrm{~V}$ (Fig. 1b) charges are well separated above a range roughly corresponding to the undepleted region $(200 \mu \mathrm{m})$ of the detector. The surprising result is that at the same threshold also a good mass separation appears for ions up to around Neon (absent at $290 \mathrm{~V})$. 


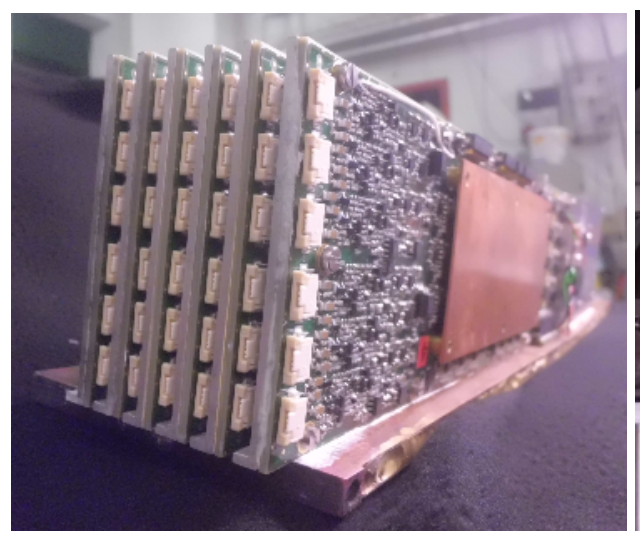

(a)

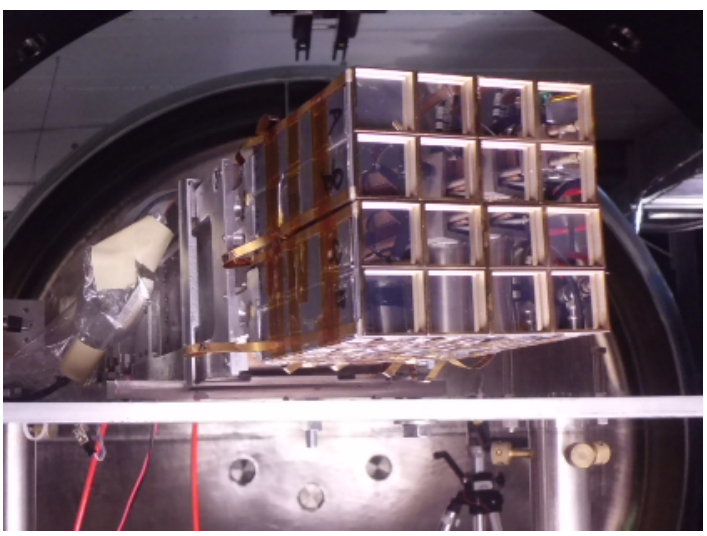

(b)

Figure 2: Left: Front view of six FEE cards mounted on the cooling copper plate. The 36 female connectors will receive the corresponding kapton strips from the detectors. Right: Picture of the first FAZIA block mounted for test purposes in the Ciclope scattering chamber at LNS, Catania.

5. Comparing the two shape variables, for partially depleted silicon, the method E vs. IMAX offers much better separation than E vs. QRT for partial depletion.

Although preliminary, these results open new possibilities. For example, depending on the experiment, one can select the bias voltage to tune the identification capability of the telescopes, in some cases preferring lower thresholds with worse isotopic separation, while privileging in other cases a good $\mathrm{Z}$ and $\mathrm{A}$ identification at the cost of higher thresholds.

\section{The demonstrator: construction and programs}

Basing on the results reported in Sect. 2), FAZIA is now constructing an array of 192 modules organized in blocks, each featuring 16 telescopes (see picture in Fig. 2b). The block is a stand-alone unit and presents original solutions to allow for vacuum operation of the FEE electronics, located next to the detectors. Long kapton strips $(16-20 \mathrm{~cm})$ connect the detectors (silicon and CsI) to the preamplifiers which are hosted on specific cards together with the ADC-FPGA stages. Each card serves 6-channels (i.e. two telescopes). Preamplifiers and digital processing electronics are thus produced on the same card, reducing noise and pick-up risk and allowing for an optimum use of the FPGA capabilities for the embedded logics. Eight FEE cards are needed for each block. They are mounted on Al shelves in a comb configuration (Fig. 2a), tightened to a single copper plate for efficient cooling. Inside the plate, several channels permit the cold water flow needed to dissipate the heat under vacuum (about $25 \mathrm{~W}$ per block). On the same copper plate, other devices are mounted for many complex functions; essentially, they transfer digitized waveforms from electric conductors to optical fibers and perform all the slow operations which are remotely controlled (test pulse generation and regulation to the preamplifiers, production, regulation of bias supply etc. etc.). The blocks, from detectors to the output board, will be supported by special arms which allow several geometries around the target, in a scattering chamber. The foreseen mounting corresponds to a $100 \mathrm{~cm}$ distance of the silicon face from the target, but angles and block assembling can be changed at will depending on the experiment. The 
angular coverage of each block is about $\pm 2.5^{\circ}$ and the angular resolution, defined by the telescope dimension, is $1.2^{\circ}$ at $100 \mathrm{~cm}$.

FAZIA foresees the use of the demonstrator for experiments with heavy ions at different european laboratories [24]. In the next future, isospin related phenomena will be studied with stable beams at intermediate energies. Two experiments have been recently approved at the LNS; they will be performed next year with an array of four complete blocks (64 telescopes). The potential of the FAZIA array in studying isospin equilibration in semiperipheral collisions has been recently demonstrated [25], although in a simplified configuration. The reaction products, (mainly) associated with the quasi-projectile decay, were measured and largely identified in mass and charge for ${ }^{86} \mathrm{Kr}+{ }^{112,124} \mathrm{Sn}$ collisions at $35 \mathrm{MeV} / \mathrm{u}$ in an experiment performed at LNS. The results, reported also at this conference [26], nicely confirm previous experimental findings [6] and evidence the occurrence of both isospin drift and isospin diffusion in semi-peripheral collisions. At the same time, our data extend the range of ions for which isotopic variables can be studied (up to $\mathrm{Z}=20$ in this case). They clearly show that with the FAZIA demonstrator good quality data will be obtained to better constrain model parameters, especially those related to the nuclear symmetry energy behaviour for nuclei in extreme conditions.

\section{Conclusions}

A modular array of $192 \mathrm{Si}-\mathrm{Si}-\mathrm{CsI}(\mathrm{Tl})$ telescopes is under construction by the european FAZIA collaboration. Thanks to an intense $R \& D$ phase, the ion identification capability of such modules has been improved both adopting specific solutions for the detectors and using fast digital electronics channels featuring high resolution. The silicons have been cut to present almost random orientation and have uniform doping homogeneity. To improve PSA, they are reverse mounted in the telescopes. Full charge separation has been obtained up to over $Z=54$ also for ions stopping in the first silicon layer via PSA, with a threshold of 2.5 (12) MeV/u for Carbon (Tin) ions. Isotopes are separated up to the iron region by the $\Delta E-E$ method for ions stopped in second silicon or in the CsI crystal. For slower particles, the PSA allows for isotopic resolution up to $\mathrm{Z} \sim 14$ with thresholds corresponding to $\sim 50 \mu \mathrm{m}$ of (fully depleted) silicon detector. Preliminary results for partially depleted detectors indicate a strong improvement of mass separation with respect to the full depletion case at the cost of somewhat higher thresholds.

The expected performances of the FAZIA array will permit to achieve high-quality results on heavyion reaction dynamics, as recently demonstrated with an experiment in which the isospin flux has been studied at intermediate bombarding energies.

\section{References}

[1] V. Baran, M. Colonna, M. Di Toro, and V. Greco, Phys. Rep.410 335 (2005)

[2] V. Baran et al., Phys. Rev. C 85054611 (2012)

[3] S. Kumar et al., Phys. Rev. C 86044616 (2012)

[4] A. McIntosh et al., Phys. Rev. C 81034603 (2010)

[5] G. Cardella et al., Phys. Rev. C 85064609 (2012)

[6] E. DeFilippo et al., Phys. Rev. C 86014610 (2012)

[7] K. Brown et al., Phys. Rev. C 87 061601R (2013)

[8] M. D’Agostino et al., Nucl. Phys. A 86147 (2011)

[9] D. Henzlova et al., Phys. Rev. C 78044616 (2008) 
[10] T. Paduszynski et al., Nucl. Instr. Meth. A 547464 (2005)

[11] T. Amorini et al., IEEE Nucl. Sci. Symp. Conf. Record N 36-4 2115 (2009)

[12] S. Wuenschel et al., Nucl. Instr. Meth. A 604578 (2009)

[13] L. Bardelli et al., Nucl. Instr. Meth. A 605353 (2009)

[14] L. Bardelli et al., Nucl. Instr. Meth. A 602501 (2009)

[15] L. Bardelli et al., Nucl. Instr. Meth. A 654272 (2011)

[16] S. Carboni et al., Nucl. Instr. Meth. A 664251 (2012)

[17] N. LeNeindre et al., Nucl. Instr. Meth. A 701145 (2013)

[18] S. Barlini et al., Nucl. Instr. Meth. A 70789 (2013)

[19] G. Pausch et al., IEEE Trans. on Nucl. Sci.44 1040 (1997)

[20] G. Pausch et al., IEEE Trans. on Nucl. Sci.43 1097 (1996)

[21] M. Alderighi et al., IEEE Trans. on Nucl. Sci.53 279 (2006)

[22] J.A. Zoutendyk and C.J. Malone, IEEE Trans. on Nucl. Sci.NS-31 1101 (1984)

[23] P. Marini et al., Eur. Phys. Jour. Web of Conf. 204003 (2010)

[24] M.F. Rivet et al., Journ. of Phys. Conf. Series 420012160 (2012)

[25] S. Barlini et al., Phys. Rev., C 87054607 (2013)

[26] S. Piantelli et al., Poster at INPC13 